\title{
Neodymium 1D systems: targeting new sources for field-induced slow magnetization relaxation
}

\author{
Amanpreet Kaur Jassal, $a$ Núria Aliaga-Alcalde, $b^{*}$ Montserrat Corbella, $c^{*}$ Daniel \\ Aravena, $d$ Eliseo Ruize*, and Geeta Hundal $a^{*}$
}

Two non-isostructural homometallic 1D Neodymium species that display field-induced slow magnetization relaxations are presented together with theoretical studies that endorse the experimental data and provide more insight on their magnetic performance. From the latest, we establish that intramolecular exchange interactions among $\mathrm{Nd}^{\mathrm{III}}$ ions for the two compounds are negligible and dipolar exchanges prevail, leading to the conclusion that, magnetically, both systems are better described as organized 1D single molecule magnets (SMMs). Our studies also suggest that $\mathrm{Nd}^{\mathrm{III}}$ ions provide great versatility toward the achievement of homometallic chains with slow relaxation of the magnetization.

Single molecule magnets (SMMs) and single chain magnets (SCMs) stand for OD and 1D coordination compounds that present slow relaxation of the magnetization and magnetic hysteresis at low temperatures. ${ }^{1}$ SMM behavior arises from the combination of large ground-state spin $S$ and zero-field splitting parameter $D$ in isolated anisotropic compounds (mono- or polynuclear systems); the slow magnetic relaxation ${ }^{2}$ in SCMs however, appears from the magnetic interactions between anisotropic repeating units along well-isolated single chains. ${ }^{3}$ Particular ratios between anisotropy and exchange interactions among the metallic centers in the chains, allow the existence of SCM behavior. ${ }^{4}$ The interesting link between SMMs and SCMs still deserves further theoretical investigation because the 1D organization of SCMs can induce a significant increase in the energy barrier for the reversal of the magnetization; ${ }^{5}$ this being the original motivation for the construction of SCMs. ${ }^{6}$

So far, many of the SCMs reported in the literature are derived from $3 d$ ions, ${ }^{7}$ mixed $3 d-4 f$ ions ${ }^{8}$ or pre-formed bridged $3 d$ SMMs. ${ }^{9}$ Much recently, homometallic $4 f \mathrm{SCMs}^{10}$ have been presented with special interest, toward the creation of new magnetic materials, ${ }^{11}$ due to the large anisotropic values of lanthanide carriers (mainly because of their strong spin-orbit contributions) and their moderate magnetic exchange couplings. ${ }^{12}$ Everything considered, homometallic $4 f$ SCMs deserve further experimental and theoretical studies. Hitherto, Dy ${ }^{\mathrm{III}}, \mathrm{Tb}^{\mathrm{III}}, \mathrm{Er}^{\mathrm{III}}$ and $\mathrm{Ho}^{\mathrm{III}}$ ions have been primarily employed ${ }^{13}$ but further understanding of the field requires a look on other $\mathrm{Ln}^{\mathrm{III}}$ ions as well, for e.g. $\mathrm{Nd}^{\mathrm{III}}$ presented here. $\mathrm{Nd}^{\mathrm{III}}$ coordination compounds
(0D or 1D) have been mostly studied because of their luminescent properties at the near-IR region ${ }^{14}$ although it has been shown that few mononuclear $\mathrm{Nd}^{\mathrm{III}}$ systems behave as SMMs. ${ }^{15}$

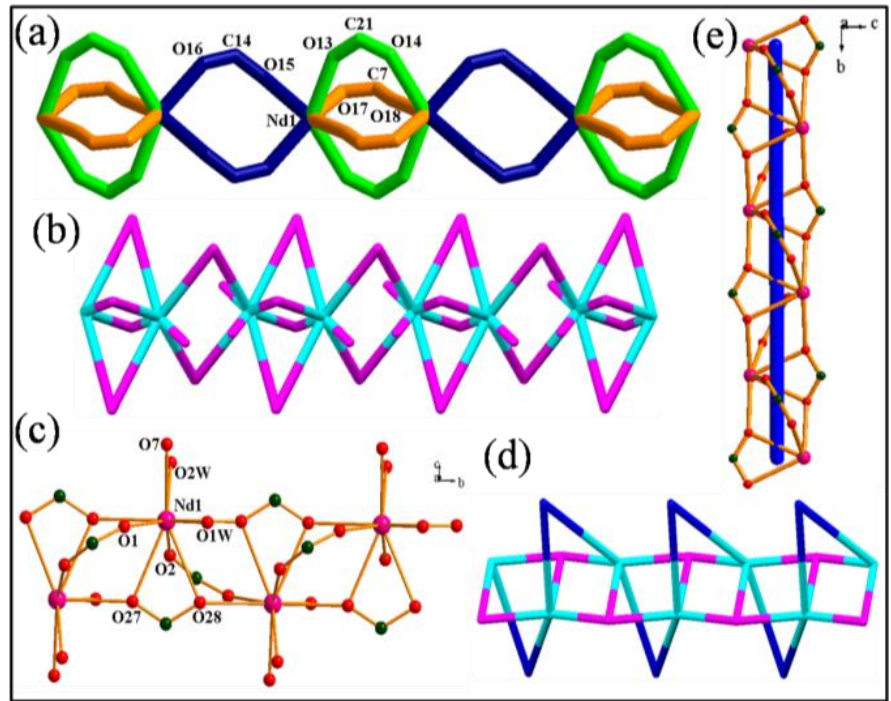

Fig. 1 (a) L1 bridged paddlewheel dimeric units (green and orange color) further bridged by L1 (blue color), forming linear tapes along $a$ axis, in 1. (b) [ $\left[\begin{array}{lll}1 & 0 & 0\end{array}\right]$ chains of 1 with 2-connected uninodal net. (c) ball-n-stick representation showing coordination environment around NdiII in 2. (d) Acetate bridged ladder along [0 $\left.\begin{array}{lll}0 & 1 & 0\end{array}\right]$ with $\mathbf{L 2}$ bridged zig-zag railing in $\mathbf{2}$. (e) Helical chain of $\mathbf{2}$ along the $b$ axis. Terminally bonded ligands are not shown in $\mathrm{d}$ and $\mathrm{e}$.

Now, we report two new non-isostructural $\mathrm{Nd}^{\mathrm{III}}$ chains, compounds 1 and 2, containing 3,5- and 2,4-dinitrobenzoic acids, $\mathbf{L 1}$ and L2, respectively. Despite two different arrangements (different coordination numbers, geometry and distances among $\mathrm{Nd}^{\mathrm{III}}$ ions, Fig. 1) both systems, 1 and 2, display field induced slow magnetization relaxations, pointing out the rich versatility

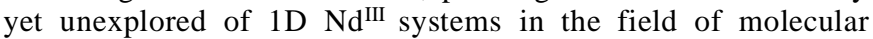


magnetism. Recently, Arauzo et al. ${ }^{16}$ accomplished the first reported $\mathrm{Nd}^{\mathrm{III}}$ homometallic chain that presents field dependence relaxation of the magnetization by the use of cyanoacetate ligands (system differs crystallographically from the two presented in this work). Here, we extend such family by the addition of $\mathbf{1}$ and $\mathbf{2}$ and provide theoretical proof disclosing the relevance of dipolar interactions in front of $\mathrm{Nd}^{\mathrm{III}} \cdot \mathrm{Nd}^{\mathrm{III}}$ exchange interactions and therefore, implying that at least $\mathbf{1}$ and $\mathbf{2}$ should be better described as organized 1D SMMs.

Compound 1, $\left\{\left[\mathrm{Nd}\left(\mu_{2}-\mathbf{L 1}\right)_{3}\left(\mathrm{H}_{2} \mathrm{O}\right)_{2}\right] . \mathrm{C}_{2} \mathrm{H}_{3} \mathrm{~N}\right\}_{\mathrm{n}}$, was achieved by mixing $\mathrm{Nd}\left(\mathrm{NO}_{3}\right)_{2} \cdot 6 \mathrm{H}_{2} \mathrm{O}$ with (L1) at room temperature meanwhile compound 2, $\left[\mathrm{Nd}\left(\mu_{2}-\mathbf{L 2}\right)(\mathbf{L 2})\left(\mathrm{CH}_{3} \mathrm{COO}\right)\left(\mathrm{H}_{2} \mathrm{O}\right)_{2}\right]_{\mathrm{n}}$, was attained after the hydrothermal reaction at $120{ }^{\circ} \mathrm{C}$ of $\mathrm{Nd}\left(\mathrm{CH}_{3} \mathrm{COO}\right)_{3} \cdot \mathrm{H}_{2} \mathrm{O}$ with (L2). Compound $\mathbf{1}$ is centrosymmetric where geometry around the $\mathrm{Nd}^{\mathrm{III}}$ ion is bicapped trigonal prismatic with six sites occupied by carboxylate oxygens of L1 and the remaining two sites by $\mathrm{H}_{2} \mathrm{O}$ molecules. Four carboxylate groups from four centrosymmetric L1 ligands coordinate two $\mathrm{Nd}^{\mathrm{III}}$ ions, in a $\mu_{2}-\kappa^{2}, \eta^{1}: \eta^{1}$ mode (mode I), with syn-syn and synanti conformation to form paddle wheel type centrosymmetric dimers (Fig. 1a). The successive dimeric units are further bridged by two more centrosymmetric L1 ligands, again in mode I, with syn-syn conformation. The dihedral angles between two Nd-OCO planes in the dimer with $\mathrm{Nd}-\mathrm{OCO}$ plane in this bridging link are $45.28(5)^{\circ}$ and 46.38(3) ${ }^{\circ}$, forming twisted ribbons (Fig. 1a) parallel to $a$ axis. The two intrachain $\mathrm{Nd} \cdots \mathrm{Nd}$ distances are 4.267 (8) $\AA$ and 5.348(7) $\AA$, respectively. Among chains, the shortest $\mathrm{Nd} \cdot \mathrm{Nd}$ distance is above $12.6 \AA$. From a topological perspective, this structure consists of chains [ $\left[\begin{array}{lll}1 & 0 & 0\end{array}\right]$ with a 2connected uninodal net (Fig. 1b).

Compound 2 belongs to chiral space group $\mathrm{P} 2_{1}$ and shows two similar but crystallographically independent molecules in the unit cell (Only one of them has been discussed here and shown in the Fig. 1). In each one of them $\mathrm{Nd}^{\mathrm{III}}$ is nine coordinated (Fig. 1c), in a trigonal prismatic tricapped geometry. Three sites are occupied by the acetate ions binding in a $\left(\mu_{2}-\kappa^{3}, \eta^{1}: \eta^{2}\right)$ mode (mode II), with a syn-syn conformation forming a ladder type architecture. Two $\mathrm{H}_{2} \mathrm{O}$ molecules and one $\mathbf{L 2}$ ligand are coordinating terminally whereas the second $\mathbf{L 2}$, in mode I, and syn-syn conformation, further links this ladder forming a spiral railing around this ladder (dihedral angle between metal-acetate plane and Nd-OCO-L2 plane is 89.74(9) ${ }^{\circ}$ ) (Fig. 1d). The resultant 1D polymer is a left handed helical chain about the $b$ axis (Fig. 1e). Finally, intermolecular interactions between $\mathrm{Nd} \cdots \mathrm{Nd}$ go over 7.7 $\AA$. The average Nd-O bond lengths, 2.4291(17) $\AA$ and 2.498 (3) $\AA$ for compounds 1 and 2 respectively, are comparable to those reported earlier for other $\mathrm{Nd}^{\mathrm{III}}-\mathrm{O}$ complexes. ${ }^{14}$ (See Fig. S1S6, Table S1-S5, ESI for details on X-ray structures).

The magnetic behavior of compounds $\mathbf{1}$ and $\mathbf{2}$ was studied by means of magnetic susceptibility experiments in the range of temperatures of $1.8-300 \mathrm{~K}$ applying 0.5 and $0.3 \mathrm{~T}$ dc fields, respectively. As shown in Fig. 2, the room temperature $\chi_{\mathrm{M}} T$ values of 1 and 2 are 1.73 and $1.69 \mathrm{~cm}^{3} \mathrm{~K} \mathrm{~mol}^{-1}$, in that order, which are only slightly higher than the expected value for an isolated $\mathrm{Nd}^{\mathrm{III}}$ ion of $1.64 \mathrm{~cm}^{3} \mathrm{~K} \mathrm{~mol}^{-1}$ (ground level $\left.{ }^{4} I_{9 / 2}\right)^{17}$ pointing out that the magnetic exchange interactions among the $\mathrm{Nd}^{\mathrm{III}}$ centers, for both chains, should be rather weak or negligible. Upon cooling, the $\chi_{\mathrm{M}} T$ values of both compounds, $\mathbf{1}$ and $\mathbf{2}$ decrease gradually, reaching the values of 0.52 and $0.79 \mathrm{~cm}^{3} \mathrm{~K}$ $\mathrm{mol}^{-1}$ at the lowest temperatures, in that order. The magnetization curves of $\mathbf{1}$ and $\mathbf{2}$ measured at $2.0 \mathrm{~K}$ are pretty similar (insets Fig. 2), showing a gradual increase from the lowest to the highest fields with the absence of plateau in both cases. Maximum values of 1.2 and $1.5 \mu_{\mathrm{B}}$ were found for $\mathbf{1}$ and $\mathbf{2}$, respectively, where the unsaturated magnetization suggests the presence of magnetic anisotropy and/or the presence of low-lying excited states that might be populated when a field is applied. ${ }^{18}$

In both systems, such magnetic response could be explained by the facts that: a) the $M_{J}$ sublevels could be split by the crystal field in the $\mathrm{Nd}^{\mathrm{III}}$ centers where, on cooling the excited $M_{J}$ sublevels, should be progressively depopulated. ${ }^{19}$ and/or b) the magnetic exchange interaction/s among neighbor metal ions in the chain. Compounds $\mathbf{1}$ and $\mathbf{2}$ present both exclusively $\mathrm{Nd}^{\mathrm{III}}$ ions attached by carboxylate bridges with syn-syn and/or syn-anti conformations that could provide antiferro- or weak antiferro/ferro-magnetic exchanges, a part from the contributions of the alkoxo groups in the case of $\mathbf{2}^{20,21}$ From an experimental point of view, it is not trivial to determine the relative nature and strength of the exchange contribution/s, as equally difficult is the analysis of the crystal field role of the ions in the net magnetic behavior. $^{22}$ The correct interpretation of these parameters and their effects requires theoretical studies, as key analyses for the proper description of the magnetic behavior. Hence, to further investigate the interplay of local crystal field and coupling effects for compounds $\mathbf{1}$ and $\mathbf{2}$, CASSCF+RASSI calculations were performed, as implemented in the MOLCAS 7.8 software package. $^{23}$
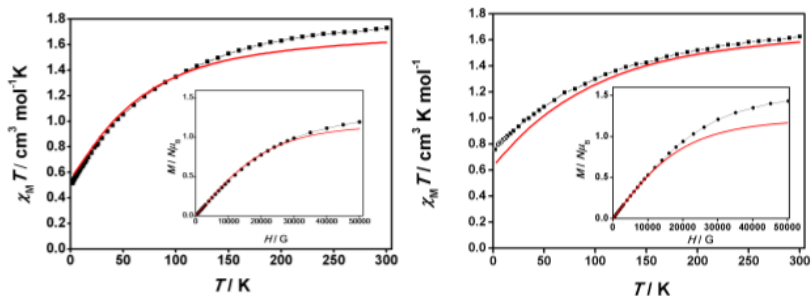

Fig. 2 (left) Plot of $\chi_{\mathrm{M}} T v s T$ for $\mathbf{1}$ (black dots correspond to the experimental measures while red line is the simulated curve using the CASSCF results for a truncated model chain using the POLY_ANISO code, see Computational Studies section). Inset: $M / \mathrm{N} \mu_{\mathrm{B}} v s H$ at $2 \mathrm{~K}$. (right) Plot of $\chi_{\mathrm{M}} T v s T$ for 2 . Inset: $M / \mathrm{N} \mu_{\mathrm{B}}$ vs $H$ at $2 \mathrm{~K}$.

The CASSCF+RASSI (or CASSCF+QDPT) approach is especially appropriate to handle the magnetic anisotropy in lanthanide systems, 24,25 as it considers the multi reference nature of the lowlying electronic energy spectrum of lanthanide-based compounds and includes the state mixing due to spin-orbit coupling, which is crucial for SMM/SCM properties. Starting from the X-ray diffraction structures, the molecular chains where truncated to monomeric models, where the immediate coordination environment of the NdIII ions was preserved. Neighbor NdIII ions were modeled as LaII $a b$ initio model potentials (truncated geometries are presented in ESI, Fig. S7). 35 quartet and 112 doublet roots were considered for the CASSCF step, while the employed ANO basis set had the following contraction patterns: $\mathrm{Nd}$ [9s8p6d4f3g2h]; O [4s3p1d]; N [4s3p21d]; C [3s2p]; H [2s]. Spin Hamiltonian parameters (such as $g$-factors) were calculated through the SINGLE_ANISO program.26

The calculated values collected in Table 1 show a large anisotropy for the two systems, $\mathbf{1}$ and $\mathbf{2}$, with a relatively low energy for the first excited state ( $77.8 \mathrm{~cm}-1$ and $87.3 \mathrm{~cm}-1$ for $\mathbf{1}$ and 2 , respectively). In Fig. 2, calculated magnetization and susceptibility curves are represented together with the experimental ones, with reasonable agreement in both cases, assuming that the exchange interaction is negligible24 and only the dipolar term is taken into account (performed with the POLY_ANISO code).27 Verification of the shape of the curve remaining constant was performed by increasing 
the number of NdIII centers (from 2 to 10 centres) in the truncated chain structure employed in the simulation. This assumption was also previously shown valid in HoIII compounds.28 The largest $\chi \mathrm{M} T$ value at low temperature for $\mathbf{2}$ in comparison with $\mathbf{1}$ is due to the larger magnetic moment of the ground state for the system $\mathbf{2}$ as reflected in the $g_{z}$ values shown in Table 1 .

Table 1 Calculated CASSCF+RASSI energies $\left(\mathrm{cm}^{-1}\right)$ and $g$-factors for the five lowest Kramers' doublets for the truncated models of compounds 1 and 2.

\begin{tabular}{c|cccc|cccc}
\hline & \multicolumn{5}{|c|}{$\mathbf{1}$} & \multicolumn{4}{c}{$\mathbf{2}$} \\
& Energy & $g_{z}$ & $g_{y}$ & $g_{x}$ & Energy & $g_{z}$ & $g_{y}$ & $g_{x}$ \\
\hline 1 & 0.0 & 3.514 & 2.012 & 1.021 & 0.0 & 4.122 & 1.730 & 0.622 \\
2 & 77.8 & 0.100 & 1.842 & 3.075 & 87.3 & 3.035 & 1.504 & 0.971 \\
3 & 171.8 & 4.037 & 1.130 & 0.106 & 188.0 & 4.415 & 0.862 & 0.297 \\
4 & 225.6 & 0.967 & 2.319 & 3.255 & 285.0 & 0.197 & 1.902 & 2.837 \\
5 & 259.3 & 3.615 & 1.973 & 1.294 & 325.8 & 0.619 & 2.881 & 3.474 \\
\hline
\end{tabular}

In addition, to investigate the magnetization dynamics, the temperature dependent alternating current (ac) susceptibility data for $\mathbf{1}$ and $\mathbf{2}$ were collected with and without applied dc fields. In the absence of a dc field, neither peaks nor frequency dependence were observed for the in-phase $\left(\chi_{M}^{\prime}\right)$ and out-ofphase $\left(\chi_{\mathrm{M}}{ }^{\prime \prime}\right)$ signals for any of the compounds under study. However, when a small dc field was applied, frequency dependence was observed for both systems due to the full or partial suppression of the quantum tunneling relaxation of the magnetization. To find the optimal dc field, parallel experiments for $\mathbf{1}$ and $\mathbf{2}$ were performed at $2.0 \mathrm{~K}$ when applying dc fields in the range of 0-0.3 T (Fig. S8) and 0-0.4 T (Fig. S9), respectively. As a result, $0.20 \mathrm{~T}$ and $0.35 \mathrm{~T}$ dc fields (those that induce the slowest relaxation) were chosen as the optimum fields for $\mathbf{1}$ and $\mathbf{2}$, in that order. Afterward, we performed dynamic ac magnetic susceptibility measurements for $\mathbf{1}$ and $\mathbf{2}$ (applying the corresponding dc magnetic field) as a function of temperature. The ac signals become frequencydependent below approx. 7.0 K for $\mathbf{1}$ and $\mathbf{2}$ and maxima peaks were observed around $5.0 \mathrm{~K}$ in both cases as well (Fig. 3). Therefore, both systems display typical SMM behaviors, in agreement with the theoretical calculations, where the relatively large transversal components of the magnetic moment $\left(g_{x}\right.$ and $g_{y}$ in Table 1) are consistent with the lack of slow relaxation at zero-field.

The ac susceptibility data plotted as Cole-Cole plots between 1.8 and 7.0 K (used $\mathrm{T}(\mathrm{K})$ : 1.8, 2.0, 2.5, 3.0, 3.5, 4.0, 4.5, 5.0, 5.5, 6.0, 6.5 and 7.0) for the two compounds show relatively symmetrical shapes (Fig. 4 top). In both cases, the fitting of the corresponding Cole-Cole data were performed with the CC-FIT program.29 From here it was found that the $\alpha$ parameters were always below 0.18 for $\mathbf{1}$ and 0.15 for 2 , in that order, indicating all together the existence in both systems of a single magnetization relaxation process with a narrow distribution of relaxation times. 30

The fitting also provided the relaxation times, $\tau^{\prime}$ s, for $\mathbf{1}$ and $\mathbf{2}$ at each temperature. By means of the Arrhenius law, the energy barrier defined as $U$ eff $/ K$ and the pre-exponential factor for the Arrhenius law $(\tau)$ ) were obtained (Fig. 4 bottom). This way, compound 1 show values of $U_{\mathrm{eff}} / K=27 \mathrm{~K}(19 \mathrm{~cm}-1)$ and $\tau 0=4.1 \cdot 10-7 \mathrm{~s}$ and compound 2 of $U_{\text {eff }} / K=29 \mathrm{~K}(20 \mathrm{~cm}-1)$ and a $\tau_{0}=3.1 \cdot 10-7 \mathrm{~s}$, in that order. Overall, these two non-isostructural, homometallic 1D systems display similar magnetic behavior which lies between the expected range for a SCM, comparable also to other $4 f$ - and $3 d-4 f$-SCMs and SMMs in the literature. 31
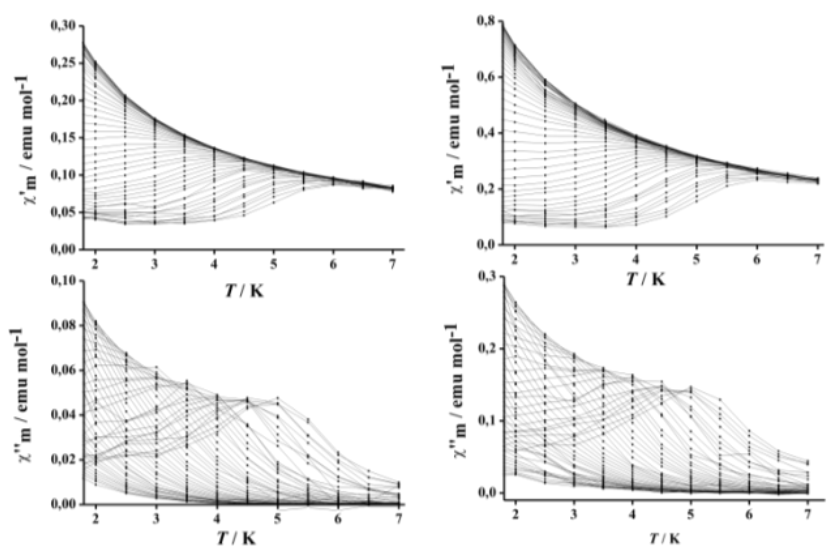

Fig. 3 In-phase (top) and out-of-phase susceptibilities (bottom) of compounds $\mathbf{1}$ (left) and $\mathbf{2}$ (right) applying an external dc magnetic field.
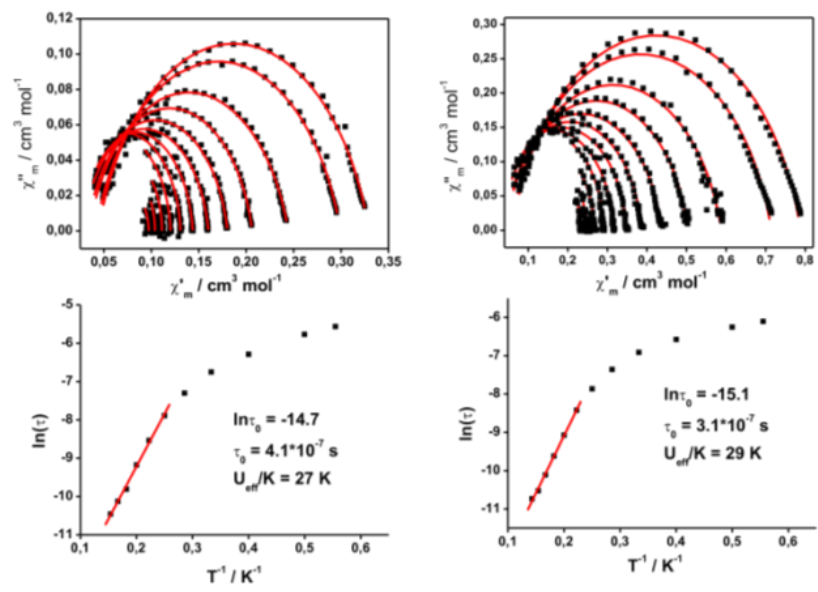

Fig. 4 (Top) Cole-Cole plots for 1 (left) and 2 (right) obtained using the ac susceptibility data applying an external dc magnetic field. (Bottom) The $\ln \tau$ vs. $T$-1 plots of 1 (left) and 2 (right). The solid lines are best fits in all the plots.

In summary, we have described two different 1D NdiII systems, compounds $\mathbf{1}$ and $\mathbf{2}$,that present slow relaxation of the magnetization by applying an external dc field, with $U_{\text {eff }} / K$ values comparable to other lanthanides SMM and SCM in the literature. These two compounds are crystallographically non-isostructural, fact that emphasizes the unexplored possibilities of NdiII systems in the field of molecular magnetism and at the same time, our theoretical studies show that the $\mathrm{Nd} \cdots \mathrm{Nd}$ exchange interactions in the two systems are negligible, been the dipolar contribution the main factor to explain the magnetic performance, behaving both as organized 1D SMMs.

A.K. Jassal thanks Department of Science and Technology for INSPIRE fellowship. G.H. thanks University Grants Commission (India) for research grant under UPE (University with potential for excellence) scheme for $G N D U$, for financial assistance. The research reported here was supported by the Spanish Ministerio de Economía y Competitividad (grants CTQ201123862-C02-0, CTQ2012-32247and CTQ2012-30662). E.R. thanks Generalitat de Catalunya for an ICREA Academia fellowship. D.A. thanks CONICYT + PAI "Concurso nacional de apoyo al retorno de investigadores/as desde el extranjero, convocatoria 2014 82140014". The authors thankfully acknowledge the computer resources, technical expertise and assistance provided by the CESCA 


\section{Notes and references}

${ }^{a}$ Department of Chemistry, Centre of Advance Studies-II, Guru Nanak Dev University, Amritsar, Punjab, India.

${ }^{b}$ ICREA (Institució Catalana de Recerca i Estudis Avançats) - ICMABCSIC (Institut de Ciència de Materials de Barcelona), Campus UAB, 08193 Bellaterra, Spain.

${ }^{c}$ Departament de Química Inorgànica and Institut de Nanociència i Nanotecnologia, Universitat de Barcelona, Martí i Franquès 1-11, 08028 Barcelona, Spain.

dDepartamento de Química de los Materiales, Facultad de Química y Biología, Universidad de Santiago de Chile (USACH), Casilla 40, Correo 33, Chile

${ }^{e}$ Departament de Química Inorgànica and Institut de Recerca de Química Teòrica i Computacional, Universitat de Barcelona, Diagonal 645, 08028 Barcelona, Spain.

$\uparrow$ This article is dedicated to Late Professor Maninder Singh Hundal.

\$ Electronic Supplementary Information (ESI) available: [X-Ray experimental details, tables of selected bond lengths ( $\mathrm{\AA}$ ) and angles (o), Important Hydrogen bond distances $(\AA)$ and angles (o), detail of Thermo gravimetric analysis and IR spectroscopy, graphs of Magnetic properties, CCDC no. 1051022 and $\mathbf{1 0 5 1 0 2 3}$ ].

1 (a) R. Gheorghe, A. M. Madalan, J.-P. Costes, W. Wernsdorfer, M Andruh, Dalton Trans., 2010, 39, 4734-4736. (b) Y. Wang, X.-L. Li, T.W. Wang, Y. Song, X.-Z. You, Inorg. Chem., 2010, 49, 969-976. (c) L. Bogani, C. Sangregorio, R. Sessoli, D. Gatteschi, Angew. Chem., Int. Ed., 2005, 44, 5817-5821. (d) K. Bernot, L. Bogani, A. Caneschi, D. Gatteschi, R. Sessoli, J. Am. Chem. Soc., 2006, 128, 7947-7956. (e) X. Wang, X. Bao, P. Xu and L. Li, Eur. J. Inorg. Chem., 2011, 3586-3591.

2 A. Caneschi, D. Gatteschi, N. Lalioti, C. Sangregorio, R. Sessoli, G. Venturi, A. Vindigni, A. Rettori, M. G. Pini, M. A. Novak, Angew. Chem., Int. Ed., 2001, 40, 1760-1763.

3 (a) G. Aromí and E. K. Brechin, Struct. Bonding, 2006, 122, 1-67. (b) D. Gatteschi, R. Sessoli, J. Villain, Molecular Nanomagnets, Oxford University Press, Oxford, 2006.

4 J. Lajzerowicz, J. J. Niez J. Phys. Lett., 1979, 40, L165-L169.

5 (a) H.-L. Suna, Z.-M. Wang, S. Gao, Coord. Chem. Rev., 2010, 254, 1081-1100. (b) R. Sessoli, A. K. Powell, Coord. Chem. Rev., 2009, 253, 2328-2341. (c) L. Bogani, A. Vindigni, R. Sessolia, D. Gatteschi, J. Mater. Chem., 2008, 18, 4750-4758. (d) R. Inglis, L. F. Jones, C. J. Milios, S. Datta, A. Collins, S. Parsons, W. Wernsdorfer, S. Hill, S. Perlepes, S. Piligkos, E. K. Brechin, Dalton Trans., 2009, 3403-3412. (e) C. Milios, S. Piligkos, E. K. Brechin, Dalton Trans., 2008, 1809-1817. (f) A. M. Ako, V. Mereacre, R. Clerac, W. Wernsdorfer, I. J. Hewitt, C. E. Anson, A. K. Powell, Chem. Commun., 2009, 544-546.

6 (a) R. Clérac, H. Miyasaka, M. Yamashita, C. Coulon J. Am. Chem. Soc., 2002, 124, 12837-12844. (b) W.-X. Zhang, R. Ishikawa, B. Breedlove, M. Yamashita, RSC Adv., 2013, 3, 3772-3798.

7 (a) X. Y. Duan, X. Cheng, J. G. Lin, S. Q. Zang, Y. Z. Li, C. J. Zhu, Q. J. Meng, Cryst. Eng. Comm., 2008, 10, 706-714. (b) S. Su, C. Qin, S. Song, Z. Guo, R. Deng, W. Chen, X. Song, S. Wang, G. Li, H. Zhang, Cryst. Eng. Comm., 2011, 13, 6057-6064.

8 (a) D. Visinescu, A. M. Madalan, M. Andruh, C. Duhayon, J.-P. Sutter, L. Ungur, W. Van den Heuvel, L. F. Chibotaru, Chem.-Eur. J., 2009, 15, 11808-11814. (b) R. Gheorghe, A. M. Madalan, J.-P. Costes, W. Wernsdorfer, M. Andruh, Dalton Trans., 2010, 39, 4734-4736.

9 H.B. Xu, B.W. Wang, F. Pan, Z.M. Wang, S. Gao, Angew. Chem., Int. Ed., 2007, 46, 7388-7392.

10 F. Luo, Z.-w. Liao, Y.-m. Song, H.-x. Huang, X.-Z. Tian, G.-M. Sun, Y. Zhu, Z.-Z. Yuan, M.-B. Luo, S.-J. Liu, W.-Y. Xu, X.-F. Feng, Dalton Trans., 2011, 40, 12651-12655.

11 Y.-G. Huang, X.-T. Wang, F.-L. Jiang, S. Gao, M.-Y. Wy, Q. Gao, W. Wei, M.-C. Hong, Chem. Eur. J. 2008, 14, 10340-10347.

12 (a) H. Ke, G.-F. Xu, Y.-N. Guo, P. Gamez, C. M. Beavers, S. J. Teatd, J. Tang, Chem. Commun., 2010, 46, 6057-6059. (b) R. Sessoli, A. K. Powell, Coord. Chem. Rev., 2009, 253, 2328-2341. (c) S. K. Langley, B. Moubaraki, C. M. Forsyth, I. A. Gass, K. S. Murray, Dalton Trans., 2010, 39, 1705-1708 (d) X. Wang, X. Bao, P. Xu, L. Li Eur. J. Inorg. Chem. 2011, 3586-3591.

13 a) N. Ishikawa, M. Sugita, W. Wernsdorfer, J. Am. Chem. Soc., 2005, 127, 3650-3651; b) N. Ishikawa, M. Sugita, W. Wernsdorfer, Angew.
Chem. Int. Ed., 2005, 44, 2931-2935; c) S.Takamatsu, T. Ishikawa, S.-y. Koshihara, N. Ishikawa, Inorg. Chem., 2007, 46, 7250-7252.

14 (a) S. Chen, R.-Q. Fan, C.-F. Sun, P. Wang, Y.-L. Yang, Q. Su, Y. Mu, Cryst. Growth Des. 2012, 12, 1337-1346 (b) F. Dai, P. Cui, F. Ye, D. Sun Cryst. Growth Des., 2010, 10, 1474-1477.

15 (a) Y.-L. Wang, Y.-L. Jiang, Z.-J. Xiahou, J.-H. Fu, Q.-Y. Liu, Dalton Trans., 2012, 41, 11428-11437. (b) J. Gu, Z. Gao, Y. Tang, Cryst. Growth Des. 2012, 12, 3312-3323.

16 A. Arauzo, A. Lazarescu, S. Shova, E. Bartolomé, R. Cases, J. Luzón, J. Bartolomé, C. Turta, Dalton Trans., 2014, 43, 12342-12356.

17 J. Rossat-Mignod, Systematics and the Properties of the Lanthanides NATO ASI Series, 1983, 109, 255-310.

18 D. Zeng, M. Ren, S.-S. Bao, L. Li, L.-M. Zheng, Chem. Commun., 2014, 50, 8356-8359.

19 D. T. Thielemann, M. Klinger, T. J. A. Wolf, Y. Lan, W. Wernsdorfer, M. Busse, P. W. Roesky, A.-N. Unterreiner, A. K. Powell, P. C. Junk, G. B. Deacon, Inorg. Chem., 2011, 50, 1199012000 .

20 H. Arora, R. Mukherjee, New J. Chem., 2010, 34, 2357-2365.

21 F. J. Kettles, V. A. Milway, F. Tuna, R. Valiente, L. H. Thomas, W. Wernsdorfer, S. T. Ochsenbein, M. Murrie, Inorg. Chem., 2014, 53, 8970-8978.

22 T. Hamamatsu, K. Yabe, M. Towatari, S. Osa, N. Matsumoto, N. Re, A. Pochaba, h. Mrozinski, J.-L. Gallani, A. Barla, P. Imperia, C. Paulsen, J.P. Kappler, Inorg. Chem., 2007, 46, 4458-4468.

23 F. Aquilante, L. De Vico, N. Ferré, G. Ghigo, P.-A. Malmqvist, P. Neogrády, T. B. Pedersen, M. Pitoňák, M. Reiher, M., B. O. Roos, B. O., L. Serrano-Andrés, M. Urban, V. Veryazov, R. Lindh, , J. Comput. Chem., 2010, 31, 224-247.

24 D. Aravena, E. Ruiz, Inorg. Chem., 2013, 52, 13770-13778.

25 S. Gómez-Coca, D. Aravena, R. Morales, E. Ruiz, Coord. Chem. Rev., 2015, 289-290, 379-392.

26 L. F. Chibotaru, ,L. Ungur J. Chem. Phys. 2012, 137, 064112-064122.

27 L. F. Chibotaru, L.,Ungur, A. Soncini Angew. Chem. Int. Ed. 2008, 47, 4126-4129.

28 J. D. Leng, J. L. Liu, W. Q. Lin, S. Gomez-Coca, D. Aravena, E. Ruiz and M. L. Tong, Chem Commun, 2013, 49, 9341-9343.

29 CC-FIT, Copyright (C) 2014 Nicholas F. Chilton.

30 Y.-N. Guo, G.-F. Xu, Y. Guo and J. Tang, Dalton Trans., 2011, 40, 9953- 9963.

31 (a) M. Menelaou, F. Ouharrou, L. Rodríguez, O. Roubeau, S. J. Teat, N. Aliaga-Alcalde, Chem. Eur. J., 2012, 18, 11545-11549. (b) M.-X. Yao, Q. Zheng, K. Qian, Y. Song, S. Gao, J.-L. Zuo, Chem. Eur. J., 2013, 19, 294-303. (c) M. A. Palacios, S. Titos-Padilla, J. Ruiz, J. M. Herrera, S. J. A. Pope, E. K. Brechin, E. Colacio, Inorg. Chem., 2014, 53, 1465-1474. 